\title{
Energiebetrachtung beim Lager- und Kommissioniervorgang
}

\author{
Energy Efficiency in the Storage and Retrieval Process
}

\author{
Meike Braun \\ Peter Linsel \\ Frank Schönung \\ Kai Furmans \\ Institut für Fördertechnik und Logistiksysteme (IFL) \\ Karlsruher Institut für Technologie (KIT)
}

$\mathbf{Z}$ iel der ganzheitlichen Betrachtung der Umweltaspekte von Fördermitteln im Lager- und Kommissioniervorgang ist über den vollständigen Lebenszyklus Aussagen über Umweltauswirkungen zu treffen. Speziell für die Nutzungsphase werden am IFL analytische Energiebedarfsmodelle für die unterschiedlichen Fördermittel entwickelt. Deren Potential besteht v. a. darin, den aktuellen Stand heutiger Energieeffizienzmaßnahmen abzubilden und das Energieeinsparpotenzial zukünftiger Energiesparmaßnahmen abzuleiten.

[Schlüsselwörter: Energieeffizienz, Umweltaspekte, ÖkodesignRichtlinie, Fördermittel, Lager- und Kommissioniervorgang, Kennzahlensystem]

A im of the consideration of the environmental aspects of material handling equipments in the storage and retrieval process is to reveal environmental impacts over the whole life cycle. Especially for the utilization phase the power demand can be described by different analytic models, which are developed at the IFL. Their potential includes all actual and energetic topics in addition to future proceedings.

[Keywords: energy efficiency, green logistics, eco-design, storage and retrieval process]

\section{Motivation}

Die Anforderungen an die Gestaltung von Fördermitteln der Intralogistik basieren auf verschiedensten Faktoren. Ausgehend von gesellschaftlichen Trends zu einem erhöhten Umweltbewusstsein, die Ausnutzung dieser Trends zu Marketingzwecken und die Verknappung von Ressourcen müssen sich die Anbieter von Intralogistiksystemen veränderten ökologischen Rahmenbedingungen zur Entwicklung, Herstellung, Vertrieb, Betrieb und Entsorgung von Fördermitteln stellen.
Insbesondere Gesetze und Regelungen, wie die Ökodesignrichtlinie der Europäischen Union [EU09a], das Energieverbrauchsrelevante-Produkte-Gesetz [EVPH08] oder unterschiedliche Normen wie die DIN EN ISO 14001 [DIN04] verstärken die Rahmenbedingungen dieser Veränderung.

Bereits im Jahr 2005 verabschiedete die Europäische Kommission die Ökodesign-Richtlinie [EU05] zur umweltgerechten Gestaltung von energiebetriebenen Produkten, z. B. elektrische Motoren im Dauerbetrieb. Eine Erweiterung der umweltgerechten Gestaltung um energieverbrauchsrelevante Produkte, wie z. B. Fenster oder Werkzeugmaschinen, fand 2009 statt [EU09a]. Relevante Angaben in Regelungen und Rahmenbedingungen zur Umwelteffizienz [Sch06] sind meist Kenngrößen wie der Umweltaspekt $\mathrm{CO}_{2}$-Ausstoß oder der Energiebedarf einzelner Produkte.

\section{UMWELTASPEKTE UND UMWELTAUSWIRKUNGEN}

Eine vollständige Übersicht der Umweltaspekte von Fördermittel in der Intralogistik wurde im IGF-Vorhaben 16973 N „Analyse und Quantifizierung von Umweltauswirkungen von Fördermitteln in der Intralogistik“ erarbeitet. Ziel des Projektes ist dabei die Identifikation, Quantifizierung, Analyse und Bewertung der Umweltauswirkungen einzelner ausgewählter Produktgruppen.

Zur Identifizierung ist ein vollständiger Katalog von Umweltauswirkungen und Umweltaspekten erstellt worden. Die Höhe der einzelnen Umweltaspekte hängt dabei von einer Vielzahl von Einflussfaktoren ab. Einflussfaktoren können z. B. die Betriebsart (wie Dauer- oder Aussetzbetrieb) oder die technische Ausstattung des Antriebsstranges (z. B. ein Antriebsstrang aus den Übertragungsgliedern Motor, Getriebe, Frequenzumrichter etc.) sein. Nach Quantifizierung der Umweltaspekte kann schließlich eine Aussage über die jeweiligen Umweltauswirkungen in abgegrenzten Systemen getroffen werden. 
Die für die Fördermittel identifizierten Umweltaspekte wurden entlang des vollständigen Produktlebenszyklus [EU09] analysiert und ermittelt, um die Beeinflussbarkeit der Höhe der Umweltaspekte durch die unterschiedlichen Lebensphasen zu analysieren.

Eine Möglichkeit die Umweltaspekte zu quantifizieren und vereinfachte Aussagen über Umweltauswirkun- gen zu treffen, liefert das EcoReport Tool [VHK05a] der Europäischen Kommission, das im Rahmen der MEEuPMethode [VHK05b] der Ökodesign-Richtlinie entwickelt wurde. Durch Angabe der verbauten Werkstoffe, deren Massen und weiterer Daten zu einzelnen Lebensphasen können Umweltauswirkungen von energiebetriebenen Produkten abgeschätzt werden.

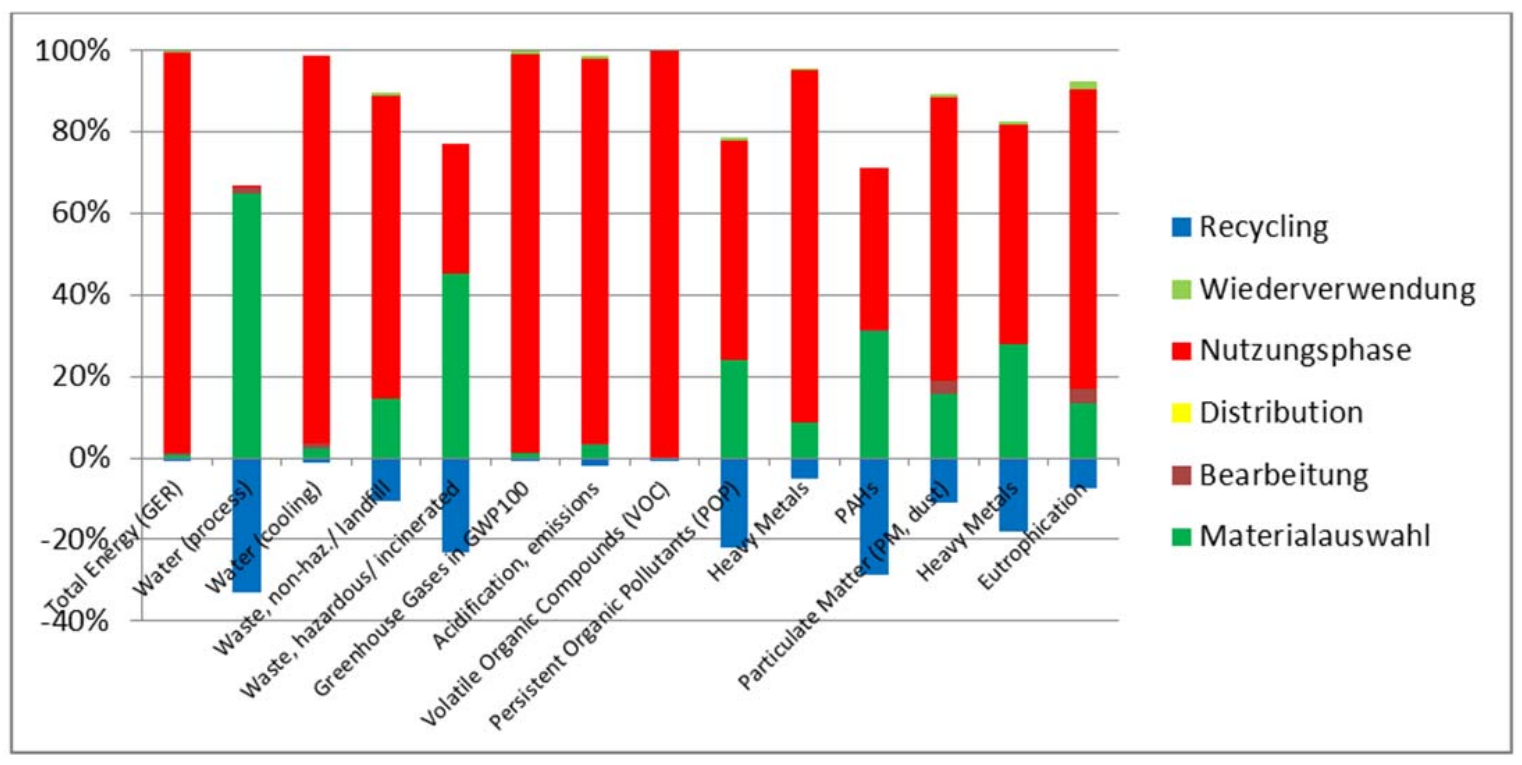

Abbildung 1. Darstellung der Ergebnisse des EcoReport-Tools am Beispiel eines Paletten-RBGs über die Lebensphasen (in Anlehnung an [Sch12a])

Abbildung 1 zeigt eine Darstellung der Ergebnisse des EcoReport-Tools am Beispiel eines Paletten-Regalbediengeräts (RBGs). Visualisiert werden einzelne Umweltaspekte, z. B. Treibhausgase im GWP $100\left(\mathrm{CO}_{2}\right.$-Ausstoß), flüchtige organische Verbindungen (VOC) oder Polycyclische aromatische Kohlenwasserstoffe (PAH), und deren Höhe prozentual aufgeteilt auf die einzelnen Lebensphasen. Daraus ist erkennbar, dass grundsätzlich die Lebensphasen „Materialauswahl“, „Nutzungsphase“ und „Recycling“ die Höhe der Umweltaspekte entscheidend beeinflussen.

Ebenfalls wird aus den Ergebnissen deutlich, dass die Beeinflussbarkeit der Umweltaspekte durch die Nutzungsphase hier am größten ist. Die Forschungsarbeit am Institut für Fördertechnik und Logistiksysteme (IFL) konzentriert sich deshalb auf eine detaillierte Betrachtung der Umweltaspekte in der Nutzungsphase. Eine genaue Analyse dieser Lebensphase ist mit Hilfe des EcoReportTools nicht möglich, da hier nur die Betrachtung des Gesamtenergiebedarfs im Fokus liegt. Um eine ausführliche Analyse und Bewertung der Nutzungsphase zu realisieren, werden am IFL analytische Modelle zur Ermittlung des Leistungsverlauf $\mathrm{P}(\mathrm{t})$ entworfen. Die Leistung wurde als Größe ausgewählt, da anhand von Leistungskennwerten einzelne Übertragungsglieder charakterisiert und modelliert werden können. Außerdem ist die Leistung eine messbare Größe, die sich durch Anwendung verschiede- ner Effizienzmaßnahmen, z. B. Einsatz von effizienten Antrieben oder von neuartigen Materialien, beeinflussen lässt. Damit kann eine erste Bewertung dieser Maßnahmen vorgenommen werden. Abzuleiten aus dem Leistungsverlauf ist der Gesamtenergiebedarf $\mathrm{E}=\int \mathrm{P}(\mathrm{t}) \mathrm{dt}$. Somit lässt sich für abgebildete Leistungsverläufe die Gesamtenergie angeben. Ausgehend vom Energiebedarf können auf Grundlage eines Stromerzeugungsmixes, z. B. für Europa (EU-25) im Jahr 2010 [Öko12], mehrere Umweltaspekte, z. B. Luftemissionen (wie $\mathrm{CO}_{2}$-Ausstoß), über bekannte Umrechnungsgrößen errechnet werden.

Mit Hilfe des EcoReport-Tools kann zwar eine vollständige Übersicht der Höhe der Umweltaspekte über alle Lebensphasen erstellt werden, die Anwendung verschiedener Effizienzmaßnahmen kann dort jedoch nicht bewertet werden. Für diese Detailbetrachtung ist die Entwicklung eigenständiger Modelle nötig.

\section{UMWELTASPEKTE UND -AUSWIRKUNGEN IM LAGER- UND KOMMISSIONIERVORGANG}

Die Prozesse eines Verteil- oder Distributionszentrums setzen sich aus „Wareneingang“, „Lager- und Kommissioniervorgang“, „Konsolidieren und Verpacken“, „Warenausgang“, sowie ggf. „Added Value“ zusammen. Da der Lager- und Kommissioniervorgang in 
fast jedem Distributionszentrum der teuerste und arbeitsintensivste Prozess ist [Mal91], [Duc05], [Kos06], fokussieren sich die Forschungsarbeiten am IFL auf diesen Prozess. Unter Lagern und Kommissionieren werden allgemein die Einlagerung, die Lagerung, die Entnahme von Waren, sowie die Entsorgung von Ladungsträgern oder Verpackungsmaterialien in einem Distributionszentrum verstanden [Wis09].

Ziel der Betrachtung ist es, die Höhe der Umweltaspekte und die Umweltauswirkungen des Lager- und Kommissioniervorgangs zu quantifizieren und zu bewerten. Dies soll vor allem durch den Aufbau der Umweltaspekt-Modelle für alle Fördermittel der Nutzungsphase, die an den Prozessen Transport, Einlagern, Lagern und der Entnahme [Wis09] beteiligt sind, erfolgen, da intralogistische Vorgänge und Materialflussprozesse in der jeweiligen technischen Umsetzung unterschiedlich gestaltet sein können.

So kann zum Beispiel ein Artikel auf einer Palette im Hochregallager gelagert und in mehreren Schritten von der Palette kommissioniert werden, andererseits kann zunächst im Wareneingang die Palette auf Kleinladungsträger vereinzelt und im AKL/ Shuttlelager gelagert und daraus kommissioniert werden.

Die beschriebene unterschiedliche Vorgehensweise ermöglicht es Unterschiede bei der Auswahl der Fördermittel und bei der Betrachtung z. B. des Energiebedarfs darzustellen. Um die Höhe des Energiebedarfs eines gesamten Lager- und Kommissioniervorgangs zu bestimmen, ist in einem ersten Schritt für jedes einzelne Fördermittel ein detailliertes Modell zu erstellen. Damit können die verschiedenen Vorgänge anhand der Kombination der unterschiedlichen Teilsystem-Modelle untersucht werden und eine Aussage über die Gesamthöhe z. B. des Energiebedarfs oder der Leistungsverläufe getroffen werden.

Am IFL wurden deshalb Teilsystem-Modelle von zehn verschiedenen Fördermitteln (Regalbediengeräte, Shuttle-Systeme, Rollenförderer, Kettenförderer, Bandförderer, Horizontale Umlaufregallager, Vertikale Umlaufregallager, Turmregallager, Teil-Automatisierte Kommissionierarbeitsplätze und Hochleistungskommissionierarbeitsplätze) erstellt. Durch Eingabe charakteristischer Größen wie der Geschwindigkeit, der Beschleunigung, der Massen oder der Verlustgrößen in die Modelle kann eine Aussage über den Gesamtenergiebedarf oder den Leistungsverlauf eines Fördermittels für eine bestimmte Tätigkeit im Lager- und Kommissioniervorgang getroffen werden. Dabei sind die Modelle nicht auf eine Geräteklasse beschränkt, sondern können individuell dem jeweiligen Gerät für verschiedene Aufgaben angepasst werden. Neben der Abbildung des Energiebedarfs heutiger Systeme kann mit Hilfe der Modelle das Energieeinsparpotential neuer Technologien und der Einfluss veränderter Abläufe abgeschätzt werden. Das Modell Regalbediengerät ermög- licht so beispielsweise die Darstellung einer Leistungsbilanz über die Anfahrt beliebiger Regalfächer, z. B. auch der charakteristischen Fächer nach der FEM-Richtlinie 9.851 eines Einzel- oder Doppelspiels [FEM03]. Damit lässt sich neben der Spielzeit, d. h. der Zeit, die zum Ausführen eines Arbeitsspiels benötigt wird, auch ein entsprechender Leistungsverlauf und Gesamtenergiebedarf angeben. Leistungsmessungen an realen Systemen dienen zur Verifizierung der Modelle und zur Ermittlung fehlender Parameter, wie z. B. der Fahrwiderstandbeiwerte.

\section{ENERGIEEFFIZIENZMAßNAHMEN}

Im Folgenden werden exemplarisch verschiedene Maßnahmen zur Erhöhung der Energieeffizienz am Beispiel von Regalbediengeräten beschrieben und erörtert. Ein wichtiges gemeinsames Ziel dieser Maßnahmen ist es, die z. B. beim Bremsen und beim Absenken der Last in der Nutzungsphase von Regalbediengeräten anfallende generatorische Energie zu nutzen. Nach heutigem Stand der Technik wird diese Energie meist über Bremswiderstände in Wärme umgewandelt und in die Umgebung abgegeben. Ein Ziel aller Maßnahmen ist die Reduzierung des Energiebedarfs und damit auch der Umweltaspekte, wie z. B. des $\mathrm{CO}_{2}$-Ausstoßes.

\subsection{Ausgangssituation AM BeisPiel EINES REGALBEDIENGERÄTS}

Zur Veranschaulichung der Energiesparmaßnahmen wird zunächst anhand eines Paletten-Regalbediengeräts die Ausgangssituation ohne Energierekuperation oder sonstige Maßnahmen betrachtet. Abbildung 2 zeigt eine Lagerfront, die ausgehend vom Ein-/ Auslagerpunkt (E/A) bei $\mathrm{x}=0, \mathrm{y}=0$ mit Hilfe eines Regalbediengerätes bedient wird. Die Länge des Lagers in x-Richtung beträgt 100 Fächer, die Höhe in y-Richtung 25 Fächer und die Lagerung findet einfachtief statt.

Die Betrachtung des Energiebedarfs erfolgt anhand der mittleren Energie der Lagerfront. Diese wurde deshalb ausgewählt, da der Mittelwert bzw. das arithmetische Mittel einer Betrachtung als statistische Maßzahl zur Kennzeichnung des Zentrums einer Verteilung angesehen werden kann. Ein erster Schritt zur Ermittlung der Verteilung ist die Betrachtung von maximalem und minimalem Energiebedarf der Lagerfront, die den Bereich aller auftretenden Energiebedarfe eingrenzen. Die Aneinanderreihung aller beobachteten Energiebedarfe stellt eine Beobachtungsreihe dar. Wird diese Beobachtungsreihe mit einer Häufigkeitsverteilung, z. B. Gleichverteilung der Anfahrhäufigkeiten, Anfahrhäufigkeitszonierungen oder Massenzonierungen, verdichtet, kann das Zentrum dieser Verteilung über den Mittelwert und damit den mittleren Energiebedarf charakterisiert werden. Im Gegensatz zur Anfahrt repräsentativer Fächer wie die der FEM-Richtlinie 9.851 [FEM03] kann mit Hilfe der Betrachtung der mittleren Energie der Regalfront eine Aussage über den 


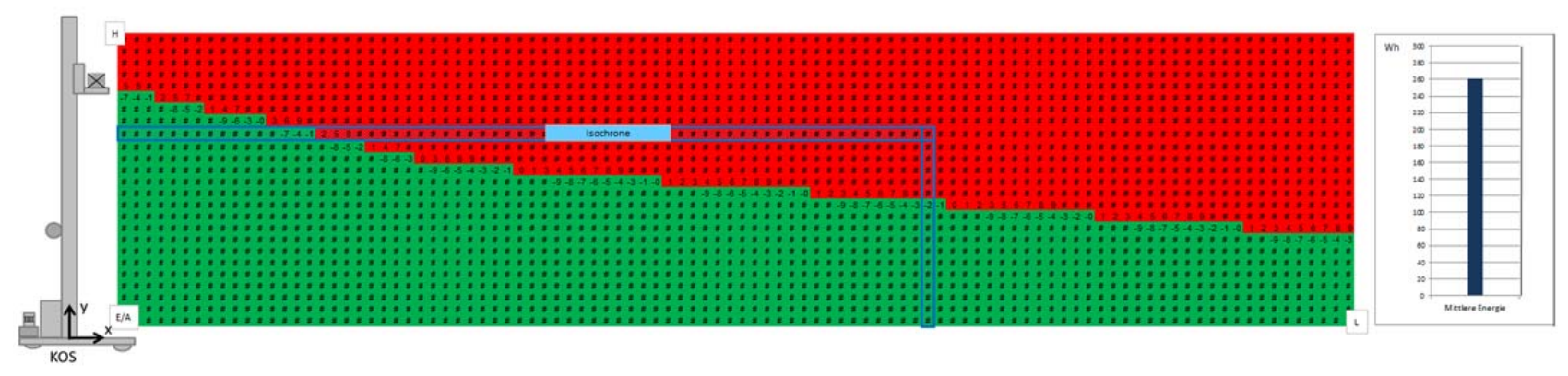

\section{Abbildung 2. Betrachtung der mittleren Energie über einer Lagerfront}

Der grüne Bereich in Abbildung 2 stellt die Fächer dar, die mit weniger als der mittleren Energie angefahren werden, während der rote Bereich die Fächer beinhaltet, deren Fahrt mehr als die mittlere Energie benötigt. Im rechten Schaubild ist die mittlere Energie der angefahrenen Fächer über der Lagerfront dargestellt.

Die mittlere Energie, die über die Lagerfront berechnet wird, setzt sich in diesem Beispiel aus Einlagerspielen zusammen. Ein Regalbediengerät fährt dabei mit einem Ladegut vom E/A ein Lagerfach an, lagert das Ladegut ein und fährt anschließend ohne Ladegut wieder zurück zum E/A. Dabei werden alle Fächer des 100x25 Lagers angefahren.

Die Grenze des grünen und roten Bereiches stellt die diskretisierte Isoenergetische der mittleren Energie dar. Sie geht durch die Fächer, die alle mit der mittleren Energie angefahren werden können [Sch12b].

Der angegebene Energiebedarf wird anhand des analytischen Umweltaspekt-Modells für das Regalbediengerät aus allen elektrischen Energien, die zum Fahren, Heben und Teleskopieren inklusive aller (Fahr-) Widerstände benötigt wird, ermittelt.

Für alle Betrachtungen in diesem Beitrag gilt über der Lagerfront eine chaotische Lagerbelegung mit einer gleichverteilten Anfahrhäufigkeit der Fächer. Die Masse des Ladegutes beträgt in diesen Fällen 1.000 kg. Es wird ein Paletten-Regalbediengerät mit ca. 20 t Gesamtgewicht als Grundlage angenommen. Die mittlere Energie der gesamten Lagerfront liegt bei $260 \mathrm{Wh}$.

\subsection{MÖGLICHKEIT 1: ENERGIEREKUPERATION IN DER NUTZUNGSPHASE (AM BEISPIEL ZWISCHENKREIS- KOPPLUNG, DOPPELSCHICHTKONDENSATOREN, ENERGIERÜCKSPEISEMODULE)}

Bei Regalbediengeräten ist jede Bewegungsrichtung mit einem Antriebsstrang, der aus Frequenzumrichter, Motor, Getriebe und weiteren Übertragungsgliedern (z. B. Seiltrommeln, Laufrädern auf Fahrschienen) besteht, ausgestattet. Bei modernen Frequenzumrichtern können die
Antriebe über einen Zwischenkreis elektrisch gekoppelt werden - die sog. Zwischenkreiskopplung -, sodass diese gemeinsame Strom- und Spannungsebene zum Energieaustausch genutzt werden kann. Generatorisch erzeugte Energie eines Antriebs kann zeitgleich in einem anderen Antrieb genutzt werden. Generatorische Energie, die auf dem Gerät nicht verbraucht wird, wird entweder als Wärme über einen Bremswiderstand abgegeben oder kann mit einer der nachfolgenden Maßnahmen genutzt werden. Zum einen kann die nicht benötigte generatorische Energie in Doppelschichtkondensatoren gespeichert werden. Diese sind aufgrund ihrer hohen Energiedichte in der Lage die hohen kurzfristig anfallenden Leistungen aufzunehmen und an die Antriebe abzugeben. Eine weitere Alternative stellt die Rückspeisung der nicht verbrauchten generatorischen Energie ins Versorgungsnetz dar. Das dafür erforderliche Rückspeisemodul bedingt allerdings ebenso wie der Einsatz von Doppelschichtkondensatoren höhere Investitionskosten und erhöhten Platzbedarf sowie erhöhtes Gewicht der Antriebstechnik. Die Zwischenkreiskopplung kann weiterhin als intelligente Zwischenkreiskopplung, bei der die Antriebsachsen gezielt koordiniert werden, ausgeführt sein [Sch09].

In Abbildung 3 sind analog zu Abbildung 2 die Energie für ein mit Zwischenkreiskopplung ausgerüstetes Regalbediengeräts (oben) und für ein mit Rückspeisemodul ausgestattetes RBG (unten) aufgezeigt. Neben der veränderten Lage der Fächer, die mit weniger (grüner Bereich) bzw. mehr (roter Bereich) als der mittleren Energie angefahren werden, ist insbesondere die geringe mittlere Energie für die Fachanfahrten (rechts) deutlich zu erkennen.

\subsection{MögLICHKEIT 2: EINSATZ ENERGIEEFFIZIENTER ANTRIEBE}

Eine der ersten Durchführungsmaßnahmen zur umweltgerechten Gestaltung von energieverbrauchsrelevanten Produkten im Zuge der Ökodesignrichtlinie [EU09a] war die Betrachtung elektrischer Motoren (mit Einschränkung auf dreiphasige $50 \mathrm{~Hz}$-Käfigläufer Induktionsmotoren, mit einer Nennausgangsleistung $P_{n}$ zwischen 

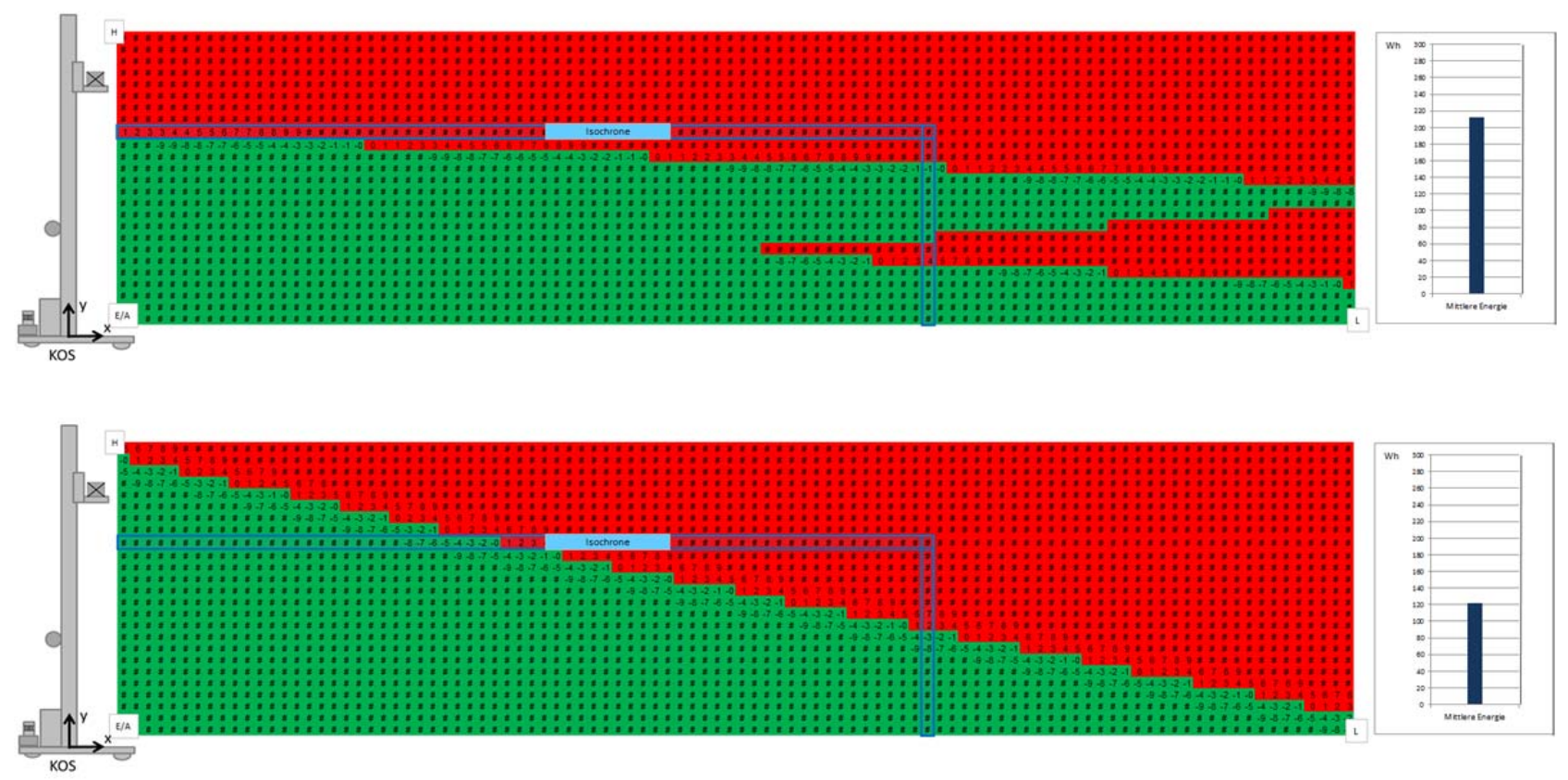

Abbildung 3. Betrachtung der mittleren Energie eines Regalbediengerätes beim Einlagereinzelspiel einer Lagerfront mit Zwischenkreiskopplung (oben) oder bei Einsatz von Rückspeisemodulen (unten)

Ziel der Durchführungsmaßnahme ist es, die NennMindesteffizienz (Wirkungsgrade) der Motoren kontinuierlich zu steigern, um dadurch z. B. Prozesse mit einem niedrigeren Energiebedarf antreiben zu können. In zeitlich festgesetzten Schritten erfolgt eine kontinuierliche Erhöhung der erforderten Effizienzniveaus [EU09b]. Unterschieden werden die einzelnen Effizienzniveaus durch die Motorenwirkungsgrade, deren Erhöhung insbesondere durch Erhöhung der Leitfähigkeit in den Rotor- und Statorleiter bzw. - wicklungen erreicht werden. Dies wird in der Regel durch Vergrößerung der Leiterquerschnitte und durch Einsatz leitfähigerer Werkstoffe ermöglicht. Beide Maßnahmen führen zu einer höheren Masse der Motoren. Aufgrund der resultierenden Erhöhung des Massenträgheitsmoments des Rotors sind nur Motoren im Dauerbetrieb (S1) von dieser Durchführungsrichtlinie betroffen. Diese Motoren finden im Lager- und Kommissioniervorgang nur im Dauerbetrieb bei Stetigförderern zum Transport der Ladeeinheiten Einsatz.

Nachfolgend werden die Umweltaspekte über den Lebensphasen am Beispiel eines Rollenförderers für Behälter über $1 \mathrm{~m}$ Förderstrecke betrachtet. Innerhalb dieser Förderstrecke ist 1 Rolle angetrieben, an die über einen Flachriemen 7 weitere mitlaufende Rollen angeschlossen sind. Als Antriebseinheit wird eine GetriebemotorKombination eingesetzt.

Aus Abbildung 4 ist erkennbar, dass im Gegensatz zur Betrachtung der Ergebnisse des EcoReport-Tools eines Paletten-Regalbediengerätes (siehe Abbildung 1) die
Höhe der Umweltaspekte schon durch die Lebensphase „Materialauswahl“ verstärkt beeinflusst wird. Die genaue Analyse der Ergebnisse, vor allem in der „Materialauswahl“, aber auch in der „Nutzungsphase“, ist Gegenstand der aktuellen Betrachtung am IFL.

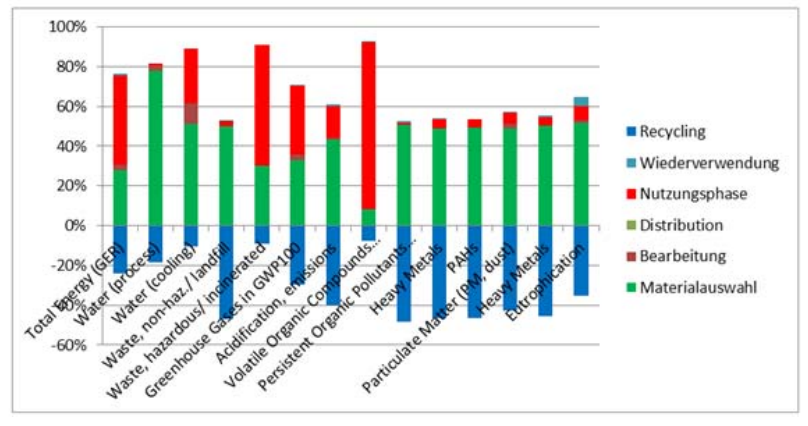

Abbildung 4. Darstellung der Ergebnisse des Eco-ReportTools eines Rollenförderers für Paletten mit externer Antriebseinheit mit Einsatz von IE2Motoren über die Lebensphasen (in Anlehnung an [Sch12a])

\subsection{MögLiCHKEIT 3: VARIATION CHARAKTERISTISCHER GRÖßEN AM BEISPIEL MASSE}

Eine weitere Möglichkeit den Energiebedarf zu minimieren ist die Variation einzelner charakteristischer Größen des RBG, d. h. die Variation von Parametern im 
analytischen Energiebedarfsmodell. Dies soll nachfolgend am Beispiel der Einflussgröße Masse aufgezeigt werden.

Das Verhältnis RBG-Gesamtmasse zu Nutzlast beträgt nach [Fur11] bis zu 9:1. Aus diesem Verhältnis erkennt man, dass eine Einsparung der Gesamtmasse ein entsprechendes Potential zur Senkung des Energiebedarfs darstellt. Diesbezüglich gibt es bereits Ansätze, durch Einsatz von Leichtbauwerkstoffen Masse einzusparen (siehe hierzu [Fur11], [Bre12] und [Ert12]).

Abbildung 5 zeigt eine Darstellung der Umweltaspekte über den gesamten Lebenszyklus unter Reduktion der Masse an Stahl und durch Einsatz von faserverstärkten Kunststoffen. In diesem Beispiel wurde der Mast eines Regalbediengerätes als glasfaserverstärkter Kunststoff ausgebildet. Daraus wird deutlich, dass die Höhe der Umweltaspekte neben der Nutzungsphase auch hier durch die Auswahl der Materialien entscheidend beeinflusst wird. Weitere Gewichtseinsparungen können durch Einsatz anderer Materialien (z. B. kohlefaserverstärkte Kunststoffe (CFK)) erzielt werden, wodurch die Energieeinsparungen (und somit auch eine Verringerung des $\mathrm{CO}_{2}-$ Ausstoßes) im Betrieb noch vergrößert werden können.

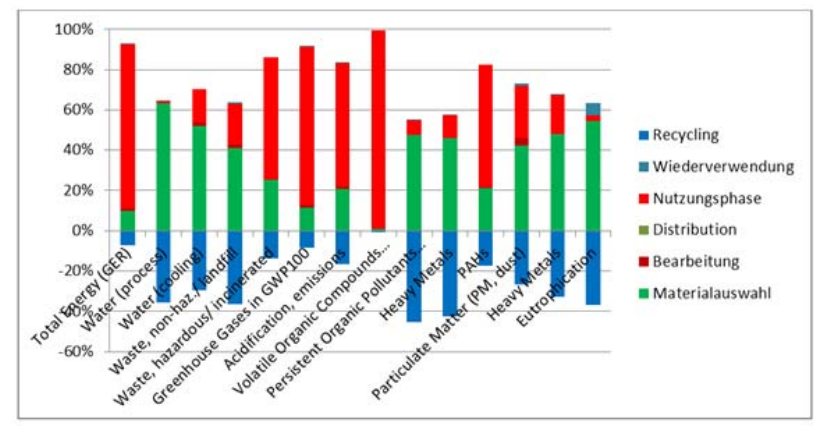

Abbildung 5. Darstellung der Ergebnisse des EcoReport Tools eines Regalbediengeräts mit Einsatz von CFK Werkstoffen über die Lebensphasen (in Anlehnung an [8])

Erfolgt eine Auswertung der Höhe der Umweltaspekte der einzelnen Materialien, so wird z. B. im Herstellungsprozess der CFK-Materialien ein Vielfaches mehr an Energie benötigt bzw. an $\mathrm{CO}_{2}$ ausgestoßen, als z. B. bei der Stahlherstellung. In der Nutzungsphase allerdings wird für die Ausführung der gleichen Aufgabe durch Verminderung des Gewichtes durch die CFK-Werkstoffe weniger Energie benötigt und die Höhe dieser Umweltaspekte damit gesenkt.

Weiterhin besteht auch noch die Herausforderung der Wirtschaftlichkeit. Durch die zurzeit höheren Herstellkosten müsste über die Betriebszeit eine signifikante Einsparung erreicht werden, damit die höheren Investitionen gerechtfertigt werden könnten. Dies verhält sich analog zu dem $\mathrm{CO}_{2}$-Ausstoß. Der Einsatz von faserverstärkten Kunststoffen bei Regalbediengeräten hätte bei derzeitigem Kostengefüge zur Folge, dass die Herstellkosten für diese Geräte höher im Vergleich zu RBGs aus Stahl bzw. mit Aluminiumanteilen liegen würden. Diese Mehrkosten müssten durch einen kostengünstigeren Betrieb wieder amortisiert werden. Somit muss, um eine Wirtschaftlichkeit des Einsatzes von neuen Materialien belegen zu können, der gesamte Produktlebenszyklus eines RBG betrachtet werden. Dies ist aktuell Bestandteil der Forschungsarbeiten am IFL.

\subsection{MÖgLICHKEIT 4: BETRIEBSWEISE / STRATEGIE - SYSTEMGEDANKE}

Ein Ansatz zur Ermöglichung einer ganzheitlichen Betrachtung der Umweltaspekte des Lager- und Kommissioniervorgangs ist es die gegenseitige Beeinflussung der einzelnen Fördermittel mit zu berücksichtigen.

Grundlage dafür ist die Betrachtung des Gesamtsystems „Technik im Lager- und Kommissioniervorgang“. Das System kann dabei in verschiedene Teilsysteme unterteilt werden, z. B. in die Teilsysteme „Fördertechnik“ oder „Lagertechnik“. Das Teilsystem „Lagertechnik“ besteht dabei aus weiteren Teilsystemen, z. B. dem Teilsystem „Regalbediengerät“. Kern der Teilsysteme sind die oben beschriebenen analytischen Energiebedarfsmodelle für die einzelnen Fördermittel. In Abbildung 6 ist schematisch eine Aufteilung des Gesamtsystems visualisiert. Kern eines jeden Teilsystems sind die eigenständigen, analytischen Umweltaspekt-Modelle.

Die Angabe des Gesamtenergiebedarfs des Lagerund Kommissioniervorgangs wird durch den modularen Aufbau der intralogistischen Vorgänge und Materialflussprozesse mit Hilfe des Systemansatzes und der Teilsystem-Modelle ermöglicht. Durch Aufrufen der einzelnen analytischen Modelle kann der Energiebedarf und der Leistungsverlauf für die unterschiedliche Ausprägung technischer Umsetzungen der intralogistischen Vorgänge und Materialflussprozesse angegeben werden.

\section{ZuSAMMENFASSUNG UND AUSBLICK}

Um den vollständigen Lager- und Kommissioniervorgang anhand von Umweltaspekten, z. B. dem $\mathrm{CO}_{2-}$ Ausstoß oder anderer ökologischer Faktoren, wie z. B. dem Energiebedarf, bewerten zu können, muss ein ganzheitlicher Ansatz gewählt werden. Grundlage dafür stellt eine Systemüberlegung dar. Die Systemgrenze wird dabei nicht um ein einzelnes Gerät, sondern um den vollständigen Vorgang gelegt, da die Aufgaben innerhalb des Lager- und Kommissioniervorgangs durch eine Vielzahl unterschiedlicher Fördermittel realisiert werden können. 


\section{Technik im Lager- und Kommissioniervorgang}

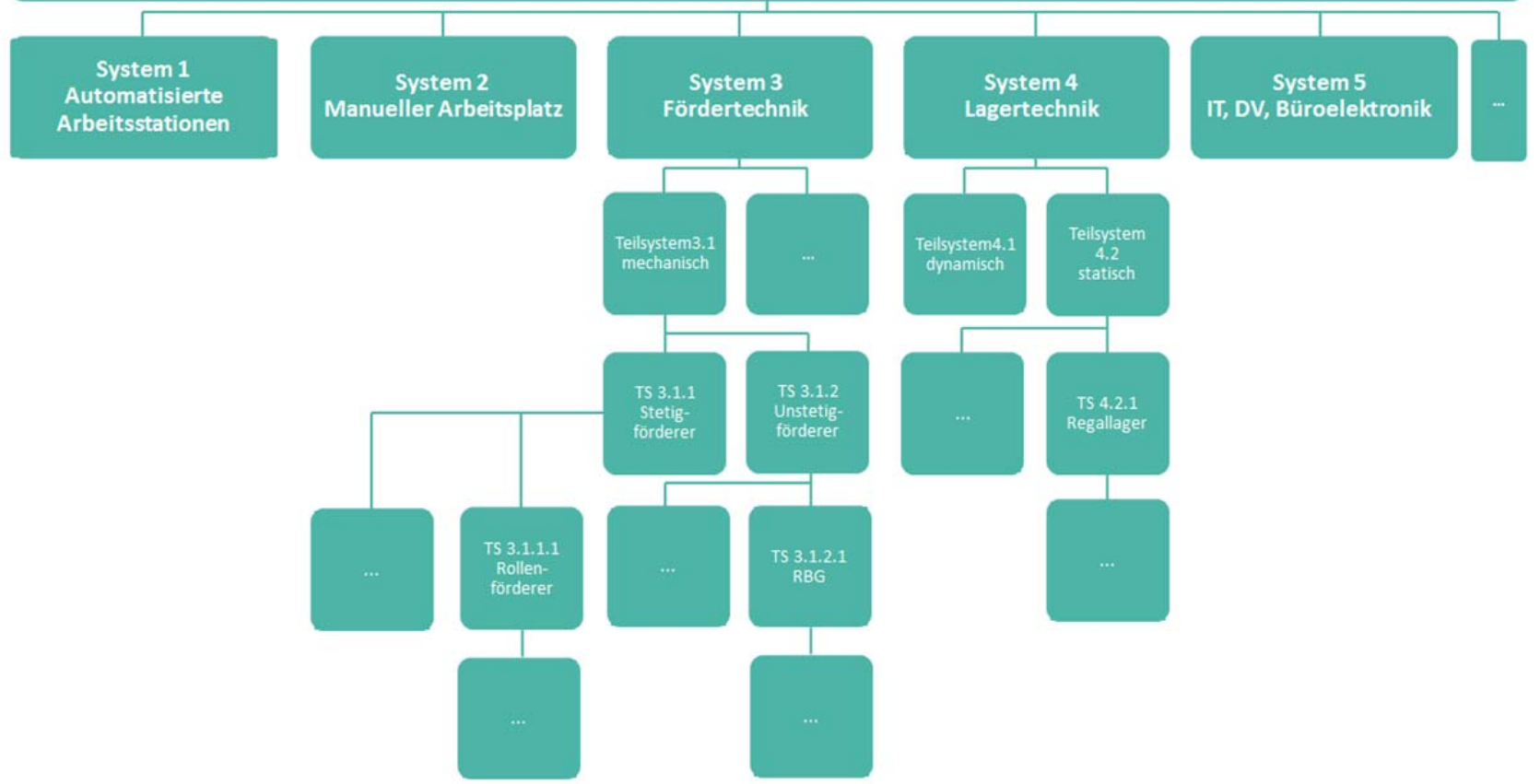

Abbildung 6. Darstellung des Systemgedankens der Technik im Lager- und Kommissioniervorgang

Eine Möglichkeit, die Höhe der Umweltaspekte zu quantifizieren, stellt das EcoReport-Tool der Europäischen Kommission dar. Aus den Ergebnissen ist erkennbar, dass vor allem durch die Nutzungsphase die Umweltaspekte der Fördermittel beeinflusst werden. Um eine detaillierte Analyse der beeinflussbaren Parameter in dieser Phase zu ermöglichen und Effizienzmaßnahmen zu bewerten, wurden am IFL analytische UmweltaspektModelle für die Nutzungsphase von zehn verschiedenen Fördermitteln erstellt. Durch den modulartigen Aufbau der intralogistischen Vorgänge kann durch Zusammenfügen der einzelnen Teilsystem-Modelle eine Gesamtaussage getroffen werden.

Die Kombination aus EcoReport-Tool und analytischen Umweltaspekt-Modellen lässt damit eine Aussage über die Höhe der Umweltaspekte in allen Lebensphasen zu.

Ebenfalls können anhand der Umweltaspekt-Modelle Energieeffizienzmaßnahmen abgeleitet werden. Die hier dargestellten Beispiele zeigen, dass eine gesamte Betrachtung aller Lebensphasen nötigt ist, um eine Bewertung der Umweltaspekte des Lager- und Kommissioniervorgangs vornehmen zu können. Insgesamt stellt die Betrachtung der Umweltaspekte sowohl für die einzelnen Fördermittel, als auch für den gesamten Lager- und Kommissioniervorgang ein herausforderndes Thema für Industrie und Forschung dar.
Neben der Entwicklung analytischer Modellen müssen auch standardisierte Möglichkeiten zur Bewertung dieser Systeme, z. B. über Kennzahlen, gegeben werden. Damit könnten unterschiedliche Intralogistiksysteme für Anwender und Anbieter dieser Systeme bewertet werden.

\section{LITERATUR}

[Bre12] Breu, Leo: Leichter, schneller, effizienter. In Materialflussspektrum - Das Fachmagazin für den Logistikleiter, 07/2012, S. 17

[DIN04] DIN EN ISO 14001:2004: Umweltmanagementsysteme - Anforderungen mit Anleitung zur Anwendung, Berlin, Beuth-Verlag 2004.

[Duc05] Le-Duc, T.: Design and control of efficient order picking processes. Dissertation, Erasmus Research Institute of Management (ERIM) RSM Erasmus Iniveversity / Rotterdam School of Economics Erasmus University, Rotterdam, Rotterdam, 2005

[Ert12] Ertl, Rainer; Günthner, Willibald A.; Fischer, Gabriel; Hahn-Woernle, Paul: Energieeffiziente Intralogistik auf Ge- 
räteebene - Beispiel Regalbediengerät In: Schenk, Michael; Zadek, Hartmut; Müller, Gerhard; Richter, Klaus; Seidel, Holger (Hrsg.): Tagungsband 17. Magdeburger Logistiktage, Otto-vonGuericke-Universität Magdeburg, Magdeburg, 27.06.2012

[EU05]

Richtlinie 2005/125/EG zur Schaffung eines Rahmens für die Festlegung von Anforderungen an die umweltgerechte Gestaltung energiebetriebener Produkte, EU-Parlament und Rat, Brüssel, 2005

[EU09a] Richtlinie 2009/125/EG zur Schaffung eines Rahmens für die Festlegung von Anforderungen an die umweltgerechte Gestaltung energieverbrauchsrelevanter Produkte, EU-Parlament und Rat, Brüssel, 2009.

[EU09b] Verordnung (EG) Nr. 640/2009 der Kommission zur Durchführung der Richtlinie 2005/32/EG des Europäischen Parlamentes und des Rats im Hinblick auf die Festlegung von Anforderungen an die umweltgerechte Gestaltung von Elektromotoren, EUParlament und Rat, Brüssel, 2009

[EVPH08] Gesetz über die umweltgerechte Gestaltung energieverbrauchsrelevanter Produkte (EnergieverbrauchsrelevanteProdukte-Gesetz - EVPH), Berlin, 2008

[FEM03] FEM 9.851: Leistungsnachweise für Regalbediengeräte Spielzeiten, Fédération Européenne de la Manutention (Europäische Vereinigung der Förderund Lagertechnik), VDMA Verlag, Frankfurt am Main, 2003

[Fur11] Furmans, Kai; Linsel, Peter: Leichtbau bei Unstetigförderern durch Einsatz moderner Werkstoffe, Beitrag zum 7. Fachkolloquium der Wissenschaftlichen Gesellschaft für Technische Logistik, 03.-04. Mai 2011, Hannover, S. 14-25

[Kos06] De Koster, René; Le-Duc, Tho; Roodbergen, Kees: Design and control of warehouse order picking: a literature review. ERIM Report Series Research in Management ERS-2006-005-LIS, 2006, S.1-30

[Lip03] Lippolt, Christian: Spielzeiten in Hochregallagern mit doppeltiefer Lagerung,
Dissertation, In: Wissenschaftliche Berichte des Institutes für Fördertechnik und Logistiksysteme der Universität Karlsruhe (TH), Band 58 , Karlsruhe, 2003

[Mal91] Malton I.: Efficient order picking - The need for it and possivle solutions. Proceeding of the 11th International Conference Automation in Warehousing 11, 1991

[Öko12] Öko-Insitut e.V., Umweltbundesamt: Prozessorienterite Basisdaten für Umweltmanagement-Instrumente (PROBAS), http://www.probas.umweltbundesamt.d e/php/index.php? am 02.08.2012

[Sch06] Schieferdecker, Bernd: Technische Tools im Industriellen Energiemanagement. In Schieferdecker, Bernd (Hrsg.): Energiemanagement Tools (2006). Berlin Heidelberg, Springer-Verlag, 2006

[Sch09] Schumacher, Meinhard: Energiesparen mit System, ETZ Elektrotechnik + Automation Heft S2/2009, VDE Verlag Berlin, S. 25-27

[Sch12a] Schischke Karsten: Energy-using Product Group Analysis - Lot 5 Machine Tools and related machinery Task 4 Report - Assessment of Base Case, Berlin, 2012

[Sch12b] Schulz, Robert; Monecke, Jörg; Zadek, Hartmut: Isoenergetische Fächer eines Automatischen Kleinteilelagers. In: Wolf-Kluthausen, Hanne (Hrsg.): Jahrbuch Logistik 2012, Korschenbroich: free beratung $\mathrm{GmbH}, 2012$

[VHK05a] VHK for European Commission: EcoReport - Excel calculation sheet, Delft 2005

[VHK05b] VHK for European Commission: MeeuP (Metholody Study Eco-design of Energy-using Products) Report, Delft 2005

[Wis09] Wisser, Jens: Der Prozess Lagern und Kommissionieren im Rahmen des DCRM, Dissertation, Wissenschaftliche Berichte des Institutes für Fördertechnik und Logistiksysteme der Universität Karlsruhe (TH), Band 72, Karlsruhe, 2009 